\title{
Postinflammatory bronchiectatic cavity observed by fibreoptic bronchoscopy
}

\author{
S. Imamura*, S. Negoro*, M. Fukuoka*, M. Kikui**
}

Postinflammatory bronchiectatic cavity observed by fibreoptic bronchoscopy. S. Imamura, S. Negoro, M. Fukuoka, M. Kikui. CERS Journals Ltd 1994.

ABSTRACT: A 55 year old female, complaining of bloody sputum and fever, was referred to our institution. The chest roentgenogram on admission revealed a large thin-walled cavitary shadow in the right upper lobe. We could clearly observe the inner surface of the postinflammatory bronchiectatic cavity in the right $B^{3}$ bronchus by fibreoptic bronchoscopy.

This is the first case in which interesting bronchoscopic findings of a bronchiectatic cavity could be directly observed.

Eur Respir J., 1994, 7, 1714-1715.

*Second Dept of Internal Medicine and **Dept of Pathology, Osaka Prefectural Habikino Hospital, Habikino City, Osaka 583, Japan.

Correspondence: S. Imamura, First Dept of Pathology, Kumamoto University School of Medicine, 2-2-1 Honjo, Kumamoto 860, Japan.

Keywords: Bronchiectatic cavity, fibreoptic bronchoscopy, postinflammation Received: September 301993

Accepted after revision April 71994
Pulmonary cavitation is frequently observed in a variety of diseases. This formation is recognized as a subsequent complication of infection, vascular disease, and cancer [1], and may often be the source of bleeding.

There have been few reports of endoscopic observation of the inner surface in a postinflammatory bronchiectatic cavity.

\section{Case report}

A 55 year old female was first referred to us for a cavitary lesion of the right lung in October, 1990. She had a history of persistent bloody sputum and cough lasting for a few days. The patient had frequently experienced these episodes for a period of 35 yrs. In 1954, she was successfully treated with antibiotics for pneumonia of the right lung. There was no history of tuberculosis on admission. She complained of bloody sputum and fever. Airway bleeding was treated with a haemostatic agent (carbazochrome sodium sulphonate), and her symptoms were abolished.

Laboratory test results, including tumour marker, were within normal limits, except for an increase in the erythrocyte sedimentation rate (ESR) $\left(42 \mathrm{~mm} \cdot \mathrm{h}^{-1}\right)$ and Creactive protein $(\mathrm{CRP})\left(7.8 \mathrm{mg} \cdot \mathrm{dl}^{-1}\right)$. A purified protein derivative skin test was positive, but sputum acid-fast stain results were negative. Results of sputum examination and cytology were normal. Arterial blood gas values revealed mild hypoxaemia $(\mathrm{pH}, 7.381$; arterial oxygen tension $\left(\mathrm{PaO}_{2}\right) 8.8 \mathrm{kPa}(60 \mathrm{mmHg})$; arterial carbon dioxide tension $\left.\left(\mathrm{PaCO}_{2}\right) 5.9 \mathrm{kPa}(44 \mathrm{mmHg})\right)$. Lung volumes and spirograms appeared normal. Electrocardiogram (ECG) also appeared normal. Chest radiograph showed a large thin-walled cavitary shadow with upper diaphragmatic movement in the right upper lobe (fig. 1).
Chest X-ray performed 35 yrs earlier was normal. No other chest radiography had been performed since. Computed tomographic scanning confirmed the presence of a cavity in the right $\mathrm{B}^{3}$ lesion and volume loss in the right middle lobe. Bronchoscopic examination revealed nothing except for the bronchiectatic cavity in the right $\mathrm{B}^{3}$ bronchus (fig. 2). Bronchoarteriography demonstrated hypervascularity around the cavitary lesion. The source of bleeding was presumed to derive from this cavity.

The patient underwent a right upper and middle bilobectomy. The right upper and middle lobes of the lung showed severe fibrous adhesion of the pleura between $\mathrm{S}^{3}$ and $\mathrm{S}^{5}$, and atelectasis in most of the middle lobe, especially in $\mathrm{S}^{5}$. The cut surfaces showed cystic and

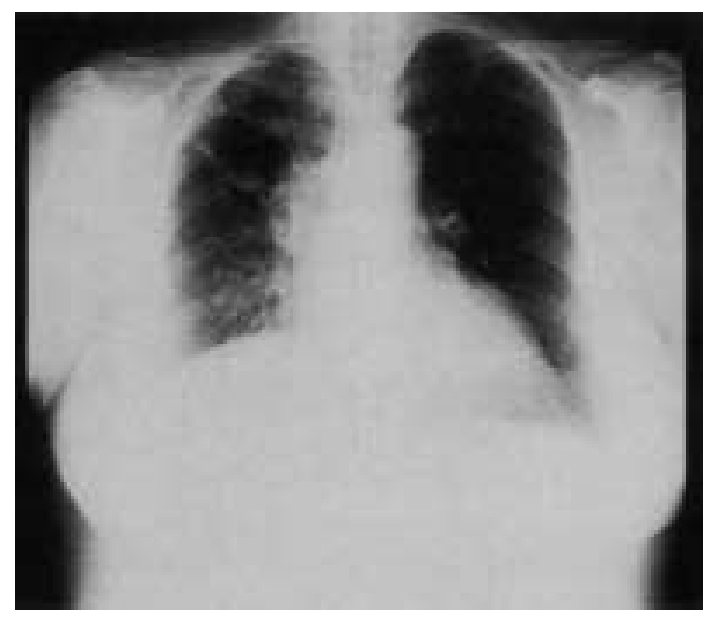

Fig. 1. - Chest roentgenogram, posteroanterior (PA) view, showing a large thin-walled cavitary shadow in the right upper lobe, with upper right diaphragmatic movement. 


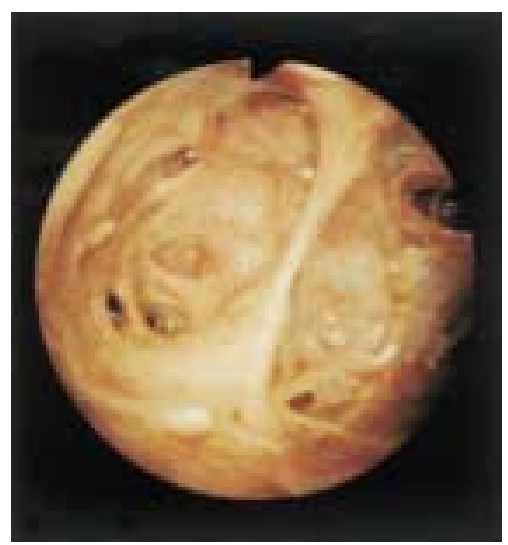

Fig. 2. - Fibreoptic bronchoscopy reveals the orifice and the inner surface of the bronchiectatic cavity of the right $\mathrm{B}^{3}$ bronchus.

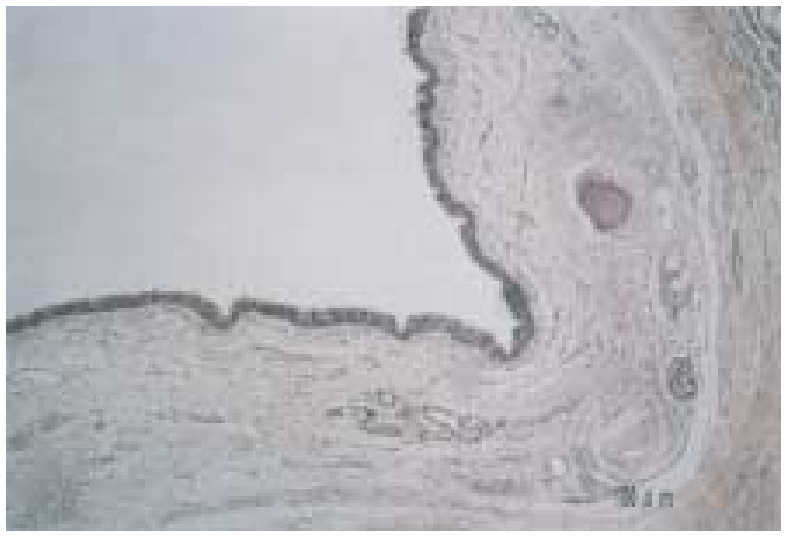

Fig. 3. - The cavity wall (right $\mathrm{B}^{3}$ ) was composed of bronchial components, such as ciliated columnar epithelium, osseous metaplastic cartilage, atrophic glands and smooth muscle fibres, all of which were accompanied by chronic inflammatory change. (Bar=100 $\mu \mathrm{m})$.

cylindrical dilatation of the bronchi. The largest bronchial dilatation, $4 \times 5 \times 3.5 \mathrm{~cm}$, was observed in $\mathrm{S}^{3}$. Its inner surface was smooth. The parenchyma around the ectasis in $\mathrm{S}^{3}$ showed fibrotic change. Microscopically, no acute inflammatory changes were noted in the resected specimen. The largest cystic lesion was lined by a ciliated columnar epithelium with an increased number of goblet cells. The cystic wall was composed of bronchial components, including smooth muscle fibres, bronchial glands and cartilage (fig. 3). Bronchial lesions similar to the largest cystic lesion were observed in the right upper lobe. These were accompanied by secondary changes, such as dilatation and fibrosis of the bronchus due to inflammation. Organized pneumonia and atelectatic change were found around the cystic lesions. There were no cystic lesions in the alveolar region. Six weeks after operation, the patient was discharged without symptoms or signs.

\section{Discussion}

Pulmonary cavity formation is often encountered clinically. Pneumonia, including lung abscess, is the major cause of its formation. With the exception of pneumonia 35 yrs earlier, the patient had no previous disease. Her cavity formation was presumed to be a subsequent complication of that pneumonia. The conditions favouring the development of pneumonia include alcoholism, diabetes mellitus, immunosuppression second-ary to various systemic diseases, poor oral hygiene, and aspiration of foreign bodies [2], but this patient had no such history. Although there have been reports of observation of the inner surface in cavities of primary lung cancer, reports on metastatic cases are few [3]. The inner surface of a postinflammatory cavity, such as this, is rarely visualized by fibreoptic bronchoscopy. Pathologically, the cavity wall was composed of bronchial components, such as smooth muscle fibres, bronchial glands and cartilage. In the alveolar region there was no cavity formation due to pneumonia or lung abscess. Therefore, the final diagnosis of this case is postpneumonic bronchiectatic cavity.

The possibility of genetic disorder must always be considered in adult patients with pulmonary disease. In cases of generalized bronchiectasis, the possible congenital aetiologies include cystic fibrosis, Kartagener's syndrome, yellow nail syndrome, combined immunodeficiency, congenital tracheobronchomegaly, and Williams-Campbell syndrome. These diseases have been found almost exclusively in infants and young children, but they have also been reported accompanying other congenital anomalies, or in patients with bronchial cartilage deficiency that produces generalized thin-walled bronchiectasis [4]. Our case exhibits a large, solitary bronchiectatic cavity and smaller bronchiectatic lesions in the right upper lobe without any generalized bronchiestasis. The cavity wall was composed of bronchial components and no deficiency or maldevelopment of bronchial cartilage was found. On examination, this patient had no apparent congenital defects.

The case presented here is noteworthy in several respects. Firstly, the patient developed bronchiectatic cavity 35 yrs after pneumonia. Secondly, microscopic observation of the resected specimen revealed that the cavity formation was not the result of acute inflammatory change. Thirdly, the bronchiectatic cavity was not the congenital but the acquired form. Finally, the cavity's inner surface was visualized by fibreoptic bronchoscopy. After undergoing surgical resection, the patient is healthy without symptoms or signs.

\section{References}

1. Reed JC. Chest radiology: plain film patterns and differential diagnoses. 3rd Edn, Chicago, Mosby-Year Book, 1991; pp. 359-399.

2. Estrera AS, Platt MR, Mills LJ, Shaw RR. Primary lung abscess. J Thorac Cardiovasc Surg 1980; 79: 275-282.

3. Yasuoka A, Maesaki S, Yamada $\mathrm{H}$, et al. A rare case of metastatic lung cancer with a cavitary lesion which was examined by bronchofiberoscopy. J Japan Soc Bronchology 1991; 13: 287-291.

4. Newman KB, Beam WR. Congenital bronchiectasis in an adult. Am J Med 1991; 91: 198-201. 